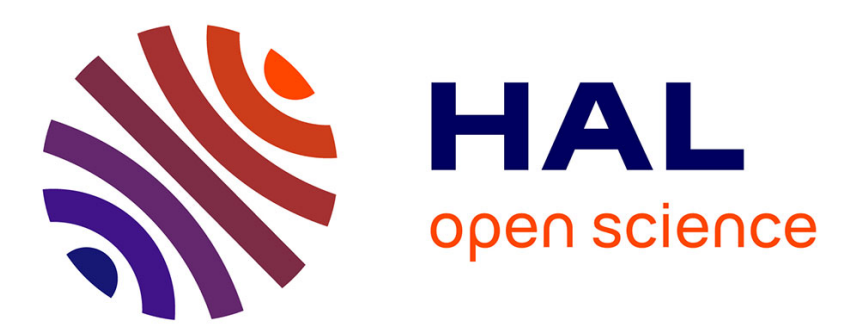

\title{
Heavy flags undergo spontaneous oscillations in flowing water
}

Michael Shelley, Nicolas Vandenberghe, Jun Zhang

\section{To cite this version:}

Michael Shelley, Nicolas Vandenberghe, Jun Zhang. Heavy flags undergo spontaneous oscillations in flowing water. Physical Review Letters, 2005, 94, pp.094302. 10.1103/PhysRevLett.94.094302 . hal-00014594

\section{HAL Id: hal-00014594 \\ https://hal.science/hal-00014594}

Submitted on 7 Sep 2015

HAL is a multi-disciplinary open access archive for the deposit and dissemination of scientific research documents, whether they are published or not. The documents may come from teaching and research institutions in France or abroad, or from public or private research centers.
L'archive ouverte pluridisciplinaire HAL, est destinée au dépôt et à la diffusion de documents scientifiques de niveau recherche, publiés ou non, émanant des établissements d'enseignement et de recherche français ou étrangers, des laboratoires publics ou privés. 


\title{
Heavy Flags Undergo Spontaneous Oscillations in Flowing Water
}

\author{
Michael Shelley, ${ }^{1}$ Nicolas Vandenberghe, ${ }^{1, *}$ and Jun Zhang ${ }^{1,2}$ \\ ${ }^{1}$ Applied Mathematics Laboratory, Courant Institute of Mathematical Sciences, New York University, \\ 251 Mercer Street, New York, New York 10012, USA \\ ${ }^{2}$ Department of Physics, New York University, 4 Washington Place, New York, New York 10003, USA
}

(Received 26 February 2004; published 9 March 2005)

\begin{abstract}
By immersing a compliant yet self-supporting sheet into flowing water, we study a heavy, streamlined, and elastic body interacting with a fluid. We find that above a critical flow velocity a sheet aligned with the flow begins to flap with a Strouhal frequency consistent with animal locomotion. This transition is subcritical. Our results agree qualitatively with a simple fluid dynamical model that predicts linear instability at a critical flow speed. Both experiment and theory emphasize the importance of body inertia in overcoming the stabilizing effects of finite rigidity and fluid drag.
\end{abstract}

DOI: 10.1103/PhysRevLett.94.094302

PACS numbers: 46.40.-f, 47.20.-k, 87.19.St

The flapping of flags in a wind is observed in everyday life, and is a prototypical instance of a streamlined and deformable body interacting with a flowing fluid. Flapping dynamics occurs also in industrial processes like paper or thin-film processing $[1,2]$ and in biological situations, like snoring [3]. Controlled experiments of the dynamics of a flag have previously been conducted in wind tunnels [2-4] and a two-dimensional analog studied in a soap-film tunnel [5]. Surprisingly, there has been to our knowledge no study of flapping dynamics conducted in water. This is, however, relevant to understanding the dynamics of towed bodies (such as underwater cable), and the general problem of coupling elasticity and inertia of a body with the dynamics of a fluid is of central interest to biological locomotion. Indeed, a waving flag can strikingly resemble the propulsive motions of a fish using undulatory propulsion $[5,6]$. The high level of performance attained by swimming fish remains a subject of active study [7], and in this context a good comprehension of the coupling between elastic and inertial body forces, and fluid forces is needed [8].

The experiments in air and soap-film flows demonstrated clearly that flapping can result from an intrinsic instability of the coupled fluid/body system alone, without the excitation of the flexible body by vortices shed from a supporting flagpole. Theoretical studies [3,9] have also extended classical work [10] on aeroelastic instabilities. These studies couple potential flow theory with the mechanics of a flag with inertia and bending rigidity, and extend Theodorsen's classical theory of wing flutter [11]; a flag has finite length and a Kutta-Zhukovski condition is imposed at its trailing edge. These studies emphasized the role of two nondimensional parameters

$$
S=m_{L} / \rho d L, \quad \hat{U}=U \sqrt{\rho d L^{3} / B},
$$

where $L$ is the length of the flag, $m_{L}$ its mass per unit length, $B$ its bending modulus, $\rho$ is the density of the fluid, $d$ is the height of the fluid layer interacting with the flag, $S$ is the ratio of the mass of the flag to that of the fluid with which it interacts, while $\hat{U}^{2}$ is the ratio of fluid kinetic energy to potential elastic energy.

Here we study the dynamics of a flexible flaglike body immersed in flowing water, fixed at its leading edge and free at its trailing end (Fig. 1). One aim is to determine whether flapping dynamics is as easily accessible in water as in air. Water is of course much denser, and if one considers the same type of sheet as used in air-typically made of fabric or plastic - the density ratio $S$ in water is about 3 orders of magnitude lower. Thus, to build a flag that flaps in water, it is essential to understand the role of this parameter in the dynamics.

To help determine how a flag might flap in water, we first use a simple model to derive analytically a stability boundary in $(S, \hat{U})$ space. Its predictions agree reasonably well with previous numerical and experimental results. The model and our preliminary experiments suggest that to flap in water, flag inertia must be sharply increased to overcome fluid drag. To avoid the complication of a heavy flag draping from its support at low flow speeds (like a "real flag"), we have designed a heavy self-supporting flag. Hence, its resting state is straight, and the fluid

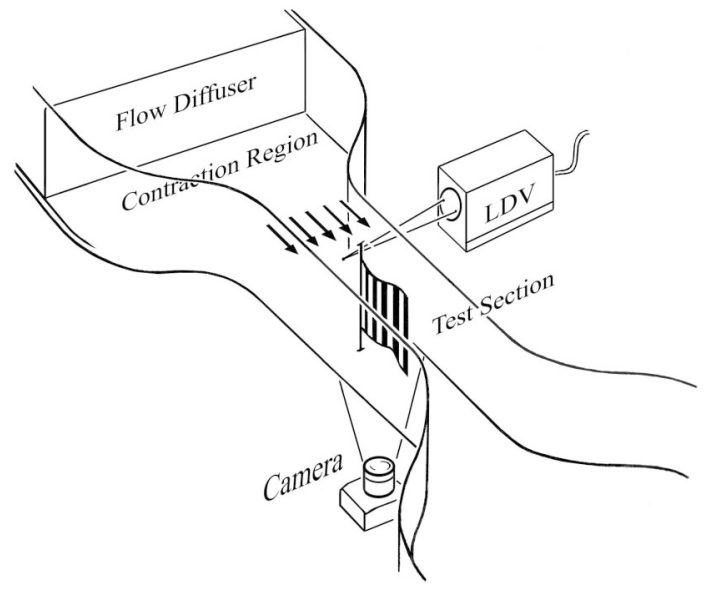

FIG. 1. Schematic of the experiment. See text for details. 
dynamics is essentially two dimensional. In agreement with previous results (and with our simplified theory) we find that, at a critical flow speed, this straight configuration becomes unstable to large amplitude flapping, the nature of which we study.

Our simple theoretical model extends Rayleigh's classical analysis [12] to include the effects of mechanical stresses at a surface of separation-the flag-whose equilibrium is represented by $L \eta(x, t)=0$. In related work, Crighton and Oswell study the interaction of a potential flow with a compliant wall of infinite extent [13]. In their analysis no external length scale is imposed and a local analysis is used to study the convective/absolute nature of the instability (see also [14]). Here, we use the length of the flag as a characteristic length of the system and examine temporal stability of perturbations on the length scale of the flag. In the equilibrium state, the speed of the flow on both sides of the flag is $U \hat{\mathbf{x}}$. A characteristic time is then $L / U$.

Following Euler-Bernoulli theory for bending beams under a small pressure load $\rho d U^{2}[p]$ and displacement $L \eta(x, t)$, a flag of mass per unit length $m_{L}$ and bending modulus $B$ satisfies the force balance

$$
S \eta_{t t}=-\hat{U}^{-2} \eta_{x x x x}+[p],
$$

where $[p]$ is the force per unit length due to the pressure jump across the flag. Here tensile forces are ignored in the force balance.

Coupling this model to the fluid dynamics yields the dispersion relation linking the frequency $\omega$ and the wave number $k$ of a perturbation of the form $\eta=\eta_{0} \exp [i(\omega t-$ $k x)][13]$

$$
(-\omega+k)^{2}+(1 / 2)\left[S|k| \omega^{2}-\hat{U}^{-2}|k|^{5}\right]=0 .
$$

This modifies the model of Crighton and Oswell to account for the flowing fluid on both sides of the surface of separation and thus there is an additional $1 / 2$ factor in the dispersion relation. For a given wave number $k$, the frequency $\omega$ satisfies the quadratic eigenvalue problem

$$
\omega=\frac{-k}{1+(1 / 2) S|k|}\left[1 \pm(1 / 2)|k|^{1 / 2} d_{k}^{1 / 2}\right]
$$

where $d_{k}=S \hat{U}^{-2}|k|^{3}+2 \hat{U}^{-2}|k|^{2}-2 S$. The system is neutrally stable if $d_{k} \geq 0$ and unstable if $d_{k}<0$.

A critical dimensionless velocity $\hat{U}_{c}$ is predicted by asking when the fundamental mode $k=2 \pi$ becomes unstable and this is determined by setting $d_{2 \pi}=\hat{U}^{-2}\left(8 \pi^{2}+\right.$ $\left.8 \pi^{3} S\right)-2 S=0$. This condition gives

$$
\hat{U}_{c}=\sqrt{\left(4 \pi^{2}+4 \pi^{3} S\right) / S} \text {. }
$$

Figure 2 shows a plot of this curve in the $S-\hat{U}$ plane. Consider varying only the velocity $U$ of the oncoming flow, as we will do in our experiment, noting that $S$ is independent of $U$. Then for $\hat{U}<\hat{U}_{c}, d_{2 \pi}$ is positive and the

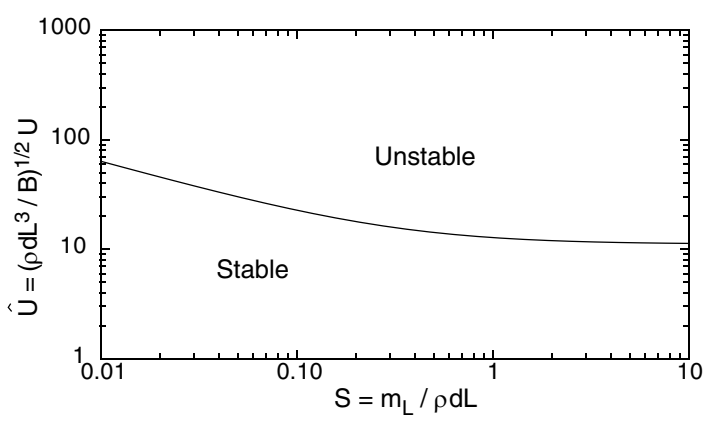

FIG. 2. The stability boundary of the straight configuration in the $S-\hat{U}$ plane as predicted by the model. The scale is such as to ease the comparison with Fig. 9 of [9].

system is stable, while for $\hat{U}>\hat{U}_{c}, d_{2 \pi}$ becomes negative and stability is lost. Note that a necessary condition for instability is that the sheet has inertia; an elastic, massless sheet supports only neutral modes (see also the simulations of [15]).

This simple model shows reasonable agreement with previous numerical and experimental results. Huang studied an aluminum foil flag in an air flow [3] with $S=$ 1.1. Our model predicts a critical speed of $9.8 \mathrm{~m} \mathrm{~s}^{-1}$ while experimentally an instability occurs at $\approx 8 \mathrm{~m} \mathrm{~s}^{-1}$. In the soap-film experiments of Zhang et al. [5], flag length is the bifurcation parameter. Reorganizing the calculation above predicts a critical length of $\sim 1 \mathrm{~cm}$, while the experiment gives $4 \mathrm{~cm}$ (there, gravity acts along the flag to also stabilize it against flapping). An overall comparison with previous theories requiring numerical computations to determine the stability boundary (see, in particular, Fig. 9 in Ref. [9]) reveals that while our model shows quantitative differences with the experimental results, the critical speed is of the same order of magnitude as those obtained from other models. Indeed our model gives a good estimate of the critical speed at high $S$, but for low $S$ the discrepancy can reach an order of magnitude, also comparable to what other models give. Note that the stability criterion based on a transition to absolute instability $[13,14]$ does not agree with previously published results (for example, the critical speed predicted for Huang's experiment is $0.088 \mathrm{~m} \mathrm{~s}^{-1}$ ).

We performed experiments to study the dynamics of a flag in a laminar water flow. The test section of the water tunnel has transparent walls, has a cross section of $15 \times$ $15 \mathrm{~cm}^{2}$, and is $45 \mathrm{~cm}$ long. The flow speed, measured by laser Doppler velocimetry (LDV; see Fig. 1), is increased from 0 up to $0.91 \mathrm{~m} \mathrm{~s}^{-1}$, with increments of $0.015 \mathrm{~m} \mathrm{~s}^{-1}$. We first used a $2.5 \mu \mathrm{m}$ thick, very flexible Mylar sheet (density $1400 \mathrm{~kg} \mathrm{~m}^{-3}, B / d=4.5 \times 10^{-10} \mathrm{~N} \mathrm{~m}$ ) of length $L=10 \mathrm{~cm}$ and height $d=5 \mathrm{~cm}$. One significant difference with an air flow is that in water the higher density yields much smaller values of $S$. For this flag, $S=3.5 \times$ $10^{-5}$ and the model predicts an instability at a flow speed of $U=1.8 \times 10^{-2} \mathrm{~m} \mathrm{~s}^{-1}$. However, we found that even with speeds of $0.9 \mathrm{~m} \mathrm{~s}^{-1}$, the flag remained flat. We soon 
realized that the inertial forces of such a light, albeit very flexible, flag were not sufficient to overcome the stabilizing effects of the fluid drag pulling on it. A simple way to estimate the effect of the drag is to compare the magnitude of tensile forces to other forces. In Eq. (2), an additional constant tension adds a term $T \eta_{x x}$. We estimate the tension, in terms of the Reynolds number Re, that results from the boundary layer drag on a flat plate [16]:

$$
T=1.33(U L / \nu)^{-1 / 2}=1.33 \operatorname{Re}_{L}^{-1 / 2} .
$$

For mode $k=2 \pi$ and speed $U=1.8 \times 10^{-2} \mathrm{~m} \mathrm{~s}^{-1}$ the stabilizing term $T k^{2}$ is 10 times higher than the term $\hat{U}^{-2} k^{4}$. This predominance of viscous drag persists for all velocities accessible in our flow tunnel.

While this suggests that the inviscid model is incomplete in this regime, and should be corrected to account for (at least) viscous drag, it also suggests that to obtain flapping, flag inertia should be much increased to reduce the relative effect of skin friction. Unfortunately, increasing either thickness or density of the material increases rigidity, and may also make the flag susceptible to draping, complicating the experiment.

We avoid these difficulties by gluing long copper strips vertically onto a Mylar sheet (left panel, Fig. 3). Carefully choosing the interstrip distance allows the flag to be pliant enough to allow flapping in one dimension, but stiff enough to avoid sagging under its own weight in the others. In the experiment described here, the Mylar sheet is $10.2 \mathrm{~cm}$ high, $7 \mathrm{~cm}$ long, and $30 \mu \mathrm{m}$ thick. Strips of copper (height $10.2 \mathrm{~cm}$, width $6.6 \mathrm{~mm}$, thickness $0.40 \mathrm{~mm}$ ) are glued back to back on the sheet, with a separation of $4.4 \mathrm{~mm}$ (of bare Mylar) between the pair. The mass of the flag is $25 \mathrm{~g}$ and the bending modulus of the Mylar sheet is $B=$ $5.9 \times 10^{-7} \mathrm{~N} \mathrm{~m}^{2}$, measured by a static bending test. The first two strips (one on each side) glued to the sheet are taped to a flagpole (a thin cylinder of diameter $1.6 \mathrm{~mm}$ ), thus ensuring clamping of the leading edge of the flag. The panel attached to the flagpole is considered inert and is not accounted for in the length and mass of the flag. Hence, there are six pairs of "free" panels.
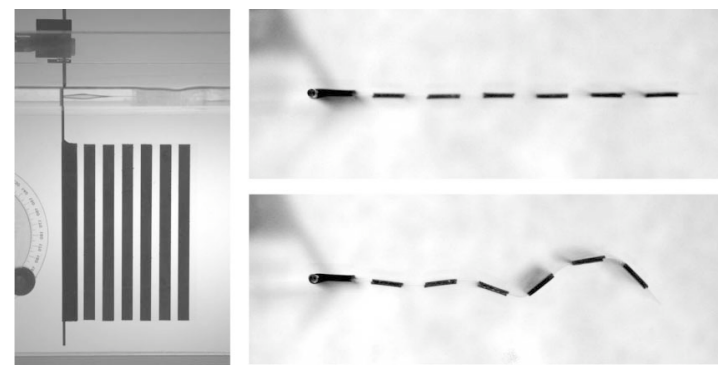

FIG. 3. The heavy flag in flowing water (left panel) and its bistable states. The laminar flow comes from the left. At flow speed $0.64 \mathrm{~m} \mathrm{~s}^{-1}$, the nonflapping (top) and flapping (bottom) states as seen from below.
For low speed flows, this "heavy flag" remains pulled out straight and steady. Crossing a critical flow speed, $U_{c}=0.81 \pm 0.015 \mathrm{~m} \mathrm{~s}^{-1}$, the flag shows a sharp transition to a flapping state of finite amplitude and frequency (Fig. 3). This bifurcation is subcritical, and at intermediate velocities, two stable states - straight or flapping - are observed (right panels, Fig. 3). As shown in Fig. 4, the amplitude of flapping increases with flow speed. The inset in Fig. 4 shows that the amplitude envelope of the flag's bending wave increases from the leading to the trailing edge. The frequency of flapping is proportional to flow speed. The resultant Strouhal number based on the flag length $S t_{L}=f L / U$ is approximately 1.2 , while the Strouhal number based on flapping amplitude increases with flow speed between 0.22 and 0.31 . Figure 4 also shows that the bending wave travels down the flag with a speed about 0.66 times the flow speed. Twisting motions of the flag were present but with amplitude of less than $3^{\circ}$.

Given the apparent resemblance between a swimming fish and the flapping of the flag, it is interesting to compare them quantitatively. One important aspect of our experiment is that the relation between frequency and velocity, as in Fig. 4, is chosen dynamically through a balance of mechanical and fluidic forces (rather than both being imposed a priori, e.g., as in [17]). The Strouhal numbers
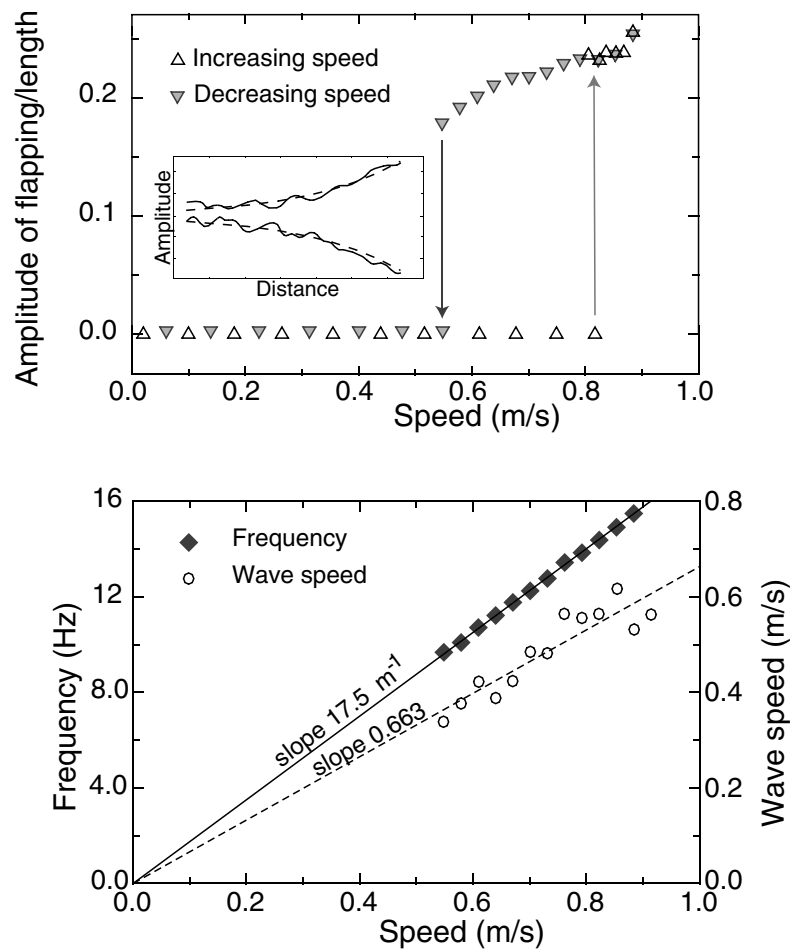

FIG. 4. Experimental results obtained by analysis of video recording at 60 frames/s. Flapping amplitude is measured as the peak-to-peak lateral displacement of the last metallic panel, normalized by the flag length. The inset shows the partial envelope (between 7 and $60 \mathrm{~mm}$ ) of the bending waves for $U=$ $0.68 \mathrm{~m} \mathrm{~s}^{-1}$. The dashed line is an exponential fit. 
observed here are also in the range observed for swimming and flying animals $[17,18]$, and are characteristic of propulsion with high power efficiency [17,19]. A related recent experiment studied the bifurcation to locomotion of a wing flapping with imposed frequency and amplitude, but free to select its speed [20]. At high flapping frequency the measured Strouhal numbers were about 0.26 , again in the same range. There are of course differences between a "passive" flag, which experiences drag, and an actively locomoting fish. One is that the bending waves traveling along the body of a swimming fish, which move on the order of 1.5 times the travel speed [21], are much faster than those observed in our experiment. This agrees with Lighthill's slender body theory of locomotion, which predicts that to produce thrust, bending waves should travel faster than the flow speed.

In the sense that our experiment shows a sharp loss of stability and a transition to flapping, it shows qualitative agreement with the hydrodynamical theory. Here the value of $S$ is 0.05 and the model gives the critical speed $U_{c}=$ $0.12 \mathrm{~m} \mathrm{~s}^{-1}$. However, to make this estimate we took the flag as homogeneous with $m_{L}=0.36 \mathrm{~kg} \mathrm{~m}^{-1}$ and $B=$ $5.9 \times 10^{-7} \mathrm{~N} \mathrm{~m}^{2}$. A better estimate of the bending modulus for this composite structure is obtained by considering the deflection of a beam made of alternating flexible and rigid parts. In that case, with a constant pressure loading, the deflection at the free end is only 0.48 times the deflection of the homogeneous flexible flag. This suggests that the equivalent bending modulus of the "composite flag" is higher and with $B=12 \times 10^{-7} \mathrm{~N} \mathrm{~m}^{2}$ we get a somewhat higher critical speed of $U=0.18 \mathrm{~m} \mathrm{~s}^{-1}$. Another factor neglected in the force balance is that our flag is self-supporting against gravity. This resistance to sagging creates a two-dimensional stress distribution in the flag that is likely modifying, and perhaps stabilizing, its bending response. A more accurate model would require a precise description of the mass and rigidity distribution along the flag. Also, this simple model does not take into account the influence of boundary conditions imposed on the flag (clamped free in experiments), the dynamics of the wake, nor the effect of skin friction. Some of these elements have been incorporated in a recent approximate model of Argentina and Mahadevan [22].

We have reported on the flapping instability of a heavy flexible sheet in a water stream. We find that viscous drag can have a strong stabilizing effect and thus can prevent "light" flags from being unstable in water. A more thorough investigation of the effect of drag on the stability may help in understanding the discrepancies observed between ideal fluid models and experiments. However, this requires a precise modeling of the near-body flow, and the propagation of waves along a body strongly modifies boundary layer flows [23]. The theoretical understanding of the nonlinear behavior-such as the subcritical bifurcation ob- served in our work and in other experiments and simulations $[5,15,24]$ - remains an open problem. Above threshold, we found that frequency is proportional to the flow speed and a Strouhal number in the range characteristic of animal locomotion. This suggests that the basic feature of the interactions between a fluid flow and an inertial body involved in efficient fish swimming may be present in this basic system. This calls for more investigation of theoretical models and for additional experiments, in particular, flow visualization to reveal the structure of the near-body flow.

We thank Steve Childress for useful conversations. This work was supported by DOE Grant No. DE-FG0288ER25033 and NSF Grant No. DMS-9980069.

*Current address: IRPHE, 49 rue F. Joliot-Curie, BP 146 13384 Marseille Cedex 13, France.

[1] Y. Chang and P. Moretti, J. Fluids Struct. 16, 989 (2002).

[2] Y. Watanabe et al., J. Fluids Struct. 16, 529 (2002).

[3] L. Huang, J. Fluids Struct. 9, 127 (1995).

[4] S. Taneda, J. Phys. Soc. Jpn. 24, 392 (1968).

[5] J. Zhang et al., Nature (London) 408, 835 (2000).

[6] U. Müller, Science 302, 1511 (2003).

[7] J. Gray, Animal Locomotion (Norton, New York, 1968); M. Triantafyllou, G. Triantafyllou, and D. Yue, Annu. Rev. Fluid Mech. 32, 33 (2000); S. Vogel, Life in Moving Fluids (Princeton University, Princeton, 1994).

[8] T. Pedley and S. Hill, J. Exp. Biol. 202, 3431 (1999).

[9] Y. Watanabe et al., J. Fluids Struct. 16, 543 (2002).

[10] Y. Fung, An Introduction to the Theory of Aeroelasticity (Dover, New York, 1955).

[11] T. Theodorsen, National Advisory Committee for Aeronautics Technical Report No. 496, 1935.

[12] L. Rayleigh, Proc. London Math. Soc. 10, 4 (1879).

[13] D. Crighton and J.E. Oswell, Philos. Trans. R. Soc. London A 335, 557 (1991).

[14] P. Brazier-Smith and J. Scott, Wave Motion 6, 547 (1984); E. de Langre, J. Sound Vib. 256, 299 (2002).

[15] L. Zhu and C. Peskin, J. Comput. Phys. 179, 452 (2002).

[16] G. Batchelor, An Introduction to Fluid Dynamics (Cambridge University, Cambridge, 1967).

[17] G. Triantafyllou, M. Triantafyllou, and M. Grosenbaugh, J. Fluids Struct. 7, 205 (1993).

[18] G. Taylor, R. Nudds, and A. Thomas, Nature (London) 425, 707 (2003).

[19] J. Anderson et al., J. Fluid Mech. 360, 41 (1998).

[20] N. Vandenberghe, J. Zhang, and S. Childress, J. Fluid Mech. 506, 147 (2004).

[21] J. Lighthill, Annu. Rev. Fluid Mech. 1, 413 (1969).

[22] M. Argentina and L. Mahadevan, Proc. Natl. Acad. Sci. U.S.A. 102, 1829 (2005).

[23] S. Taneda and Y. Tomonari, J. Phys. Soc. Jpn. 36, 1683 (1974); M. Wolfgang et al., J. Exp. Biol. 202, 2303 (1999).

[24] D. Tang, H. Yamamoto, and E. Dowell, J. Fluids Struct. 17, 225 (2003). 